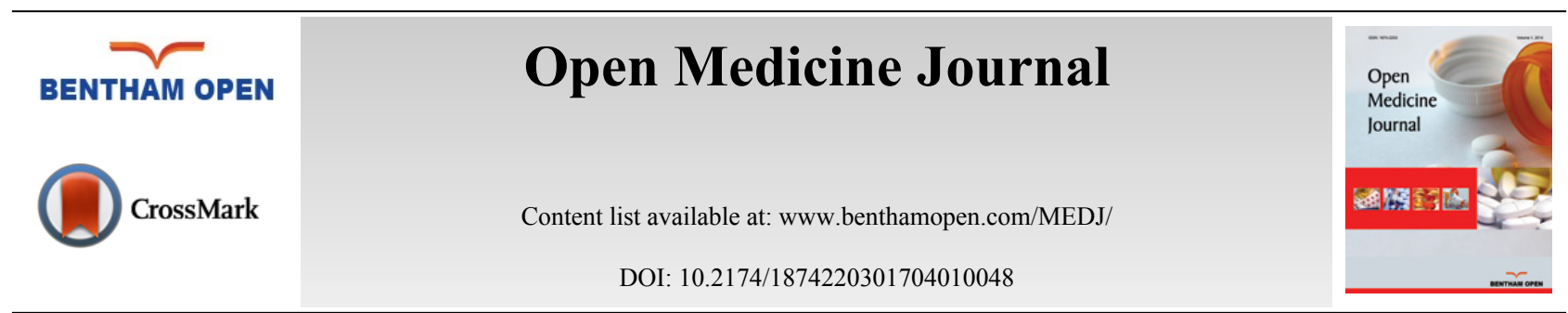

REVIEW ARTICLE

\title{
Ethics in Quality Improvement with in Health Care - Focusing on the Improvement
}

\author{
Lars Sandman ${ }^{*}$ \\ Department of Medical and Health Sciences, Linköping University, 58183 Linköping, Sweden
}

Received: December 13, 2016

Revised: April 20, 2017

Accepted: July 04, 2017

\begin{abstract}
:
Background:

Health-care is permeated with ethical values and norms and so there are ethical implications to all interventions which changes practice, and this includes quality improvement (QI). The interest for ethics in QI so far has not had an explicit focus on the ethics of the actual improvement. Contrasting this with health technology assessment (HTA), we there find an almost exclusive interest in the ethics of the developed technology, and less regard for the other ethical aspects related to development, evaluation and implementation of the technology.
\end{abstract}

\section{Method:}

We identify how a research ethics perspective on QI differs from the ethics of the actual improvement or change. It will be argued that the field of ethics in QI can benefit from the development of ethics within HTA by raising awareness of the need to ethically assess the actual improvement or change, and by using models for this found within HTA. For an ethical analysis of quality improvement, elderly care in the Swedish context will be given as a tentative example.

\section{Conclusion:}

The existing frameworks for ethical assessment within HTA need further development for use within QI. In essence, the introduction of new technologies within health-care could be viewed as a quality improvement, where considerations generally acknowledged within QI are relevant to consider in relation to all potential technologies to be used within health-care.

\section{INTRODUCTION AND AIM}

Since health-care is permeated by ethical values and norms, all interventions to change its practice will have ethical implications and thus, these ethical implications should be considered ${ }^{1}$. This is true also for quality improvement, defined as "systematic, data-guided activities designed to bring about immediate, positive changes in the delivery of health care" [1]. An important input to the ethical discussion concerning QI was the Hastings Center report The Ethics of Using QI Methods to Improve Health Care Quality and Safety [1] and its follow up in the edited book Health Care Quality Improvement: Ethical and Regulatory Issues [1]. In these publications the focus was on ethical responsibility for organizations, managers, professionals and patients to be involved in QI, on the ethical aspects related to: "social or scientific value, scientific validity, fair subject selection, favourable risk/benefit ratio, respect for potential and enrolled subjects, informed consent" [1]; and following this, also how ethical oversight of QI should take place (e.g. using institutional review board systems or not etc.). The resulting academic discussion of ethics in QI confirms this focus [2 7]. What is missing in this picture is both a more explicit ethical assessment of the actual improvement implied by QI

* Address correspondence to this author at the Department of Medical and Health Sciences, Linköping University, 58183 Linköping, Sweden; Tel: +46 (0) 732 065818; E-mail: lars.sandman@liu.se 
and also on ethical aspects related to the more full scale implementation of the QI. Contrasting this with another field of health-care or health-care decision-making, health technology assessment (HTA), we there find an almost exclusive interest in the ethics of the developed technology, and less regard for the ethics of developing and evaluating the technology and also for ethical issues surrounding the actual implementation of technology in health-care [8]. In this article it will be argued that the field of ethics in QI can benefit from the development of ethics within HTA by raising awareness of the need to ethically assess the actual improvement or change, and by using models for this found within HTA. Still, it is important to emphasize that on top of this a more explicit discussion of the ethics of implementation is also needed to arrive at a more complete picture of the ethics of QI. However, implementation aspects will not be addressed in this paper (more than in passing).

This beneficial impact will go both ways, and the field of ethical assessment within HTA can likewise benefit by taking insights from QI into account (even though the focus in this article will be on QI). The beneficial impact from developments within HTA will be illustrated by applying a (somewhat developed) model for ethical assessment within HTA on a potential QI-project or activity.

\section{A SHORT NOTE ON QI}

Given the definition of QI above, it will be assumed that quality encompass the aspects stipulated in Institute of Medicine influential report Crossing the Quality Chasm [9]. That is, safety, effectiveness, patient (or person) centeredness, timeliness, equity and efficiency. Incorporating the aspect of efficiency implies that also QI that "only" involves organizational changes or changes in professional work to improve efficiency that does not actually affect patient care ${ }^{2}$ will be included within the QI-concept. From an ethical perspective this is essential, both since we then need to consider ethical aspects of changes, even though they do not have a direct impact on patient care, but also since such a systems approach can also have consequences for how to view ethical assessment or oversight within QI.

\section{HEALTH TECHNOLOGY ASSESSMENT (HTA)}

During the last 20-30 years the field of health technology assessment (HTA) have developed and spread rapidly around the world [10] and a large amount of countries have agencies for doing HTAs when introducing new technology within health-care (and also for assessing or re-assessing old technologies). Since the more formal establishment of HTA, analysis of ethical aspects of introducing a technology is part of the modern definition of HTA [11]. For the last 10 years the ethics assessment within HTA have been developed in an unprecedented way resulting in formalized approaches to ethical analysis of new technologies, academic discussion on different aspects of ethics assessments within HTA, publications of ethical analyses of different technologies etc [12, 13]. Within HTA, "technology" is understood in a broad sense including pharmaceuticals, surgical interventions, nursing interventions, dietary recommendations, training programs etc. However, also organizational interventions or changes might be the object of assessment (i.e. be included under the concept of technology), e.g. how to organize care for the multi-diseased and vulnerable old people [14].

Within the HTA-community, there are several tools for identifying and analysing relevant ethical issues. On an international level the EUneHTA core model and its ethics part is one of the most well established [8]. However, given its rather abstract approach, within different national contexts, this model has been adapted for a better fit to national health-care legislation and preconditions. In this article a framework developed for the Swedish health-care context will be the point of departure [15].

At this point the difference between QI and HTA needs some attention. HTA generally evaluates technologies unrelated to a specific clinical context, even if general specifications of the relevant clinical context or preconditions for its use might be listed. This also implies that the ethical assessment might remain quite open in character. It can identify a certain ethical problem or, vice versa, find the technology ethically acceptable, given that the clinical context looks a specific way. QI, on the other hand, takes place in a concrete clinical context, where any relevant characteristics can be identified. Hence, the ethical assessment has the potential to become more definite in its conclusions, and can also provide more concrete guidance as to how the concrete context should be adapted to fulfil ethical requirements in

\footnotetext{
${ }^{1}$ In this context "ethics" is given a broad definition according to which it is related to anything that has value in a more fundamental sense to sentient beings (humans and other sentient beings). In health-care the ethical values normally include aspects like: the benefits (and corresponding burdens) of treatment and care; dignity, autonomy, integrity and privacy, justice, equality. This implies that derived concepts like "ethical responsibility" means responsibility that can be motivated by reference to these values.
} 
${ }^{2}$ Talking about patient care includes also care for family or significant others.

relation to the improvement. But it also means that in QI we need to be observant on unique factors in the clinical context that will have an impact on our assessment that we often can and will ignore in the HTA.

\section{WHAT IS MISSING IN THE CURRENT ETHICS APPROACH TO QI?}

Taking as departure the report from Hastings Center on the ethics of QI we can identify how the current ethics perspective on QI differs from looking at the ethics of the actual improvement or change. Substantially, disregarding their discussion on ethics review or oversight, they focus on six general areas.

Within the areas social or scientific value and favourable risk/benefit ratio the focus is on benefits and burdens or risks for participants (both patients and professionals). Possible burdens to be observant upon are: " Burdens can take the form of direct physical harms, mental and psychological harms, "hassle" harms such as time consumed in completing surveys or submitting to extra clinical or administrative procedures, or harms related to loss of privacy and confidentiality [1]. This could be interpreted as covering both the actual change or improvement as well as the "extra" QI-activities to assess whether the change actually did result in an improvement. The reference to "hassle harms" indicate a focus on the latter. However, the change or improvement can have more wide ranging ethical implications for all concerned parties if the QI is transformed into standard care.

The area fair subject selection focuses both on who gets involved in the QI-activities, but also who will benefit from them in the long run. Hence, it is both about QI-activities but also about the access to the actual improvement.

Respect of potential and enrolled subjects and Informed consent are overlapping and covers questions about information and informed consent, privacy and confidentiality. Once again this could cover both QI-activities and the improvement, but in the report the discussion is clearly focused on the participants involved in specific QI-activities. Here, the authors emphasize an interesting difference in relation to informed consent in relation to research, that unless we are dealing with a particularly risky QI, informed consent from participants is not required (since it might be viewed as an ethical responsibility).

To emphasize, it is important to consider these ethical aspects of QI, hence when contrasting this approach with models for ethically assessing technologies within HTA - we are looking for complementary aspects to consider. Consider the above-mentioned Swedish framework developed to identify and assess ethical aspects for health-care technologies (broadly understood also including organizational changes). In this we find 12 question-areas organized in four different sections: Impact on health, Specific ethical values and norms, Structural factors with ethical implications and Long-term effects [15].

\subsection{Impact on Health:}

1. How does the technology affect the health and quality of life of the patient?

2. Are there any knowledge gaps concerning these effects, and are there any ethical or methodological obstacles to explore these?

3. How severe is the condition, to be treated by the technology? The more severe it is, the greater the reason to use the technology.

4. How does the technology affect third parties' health (family and other stakeholders)?

\subsection{Specific Ethical Values and Norms:}

1. How does the technology affect patient and third party equality and equity?

2. How does the technology relate to patient and third party autonomy?

3. How does the technology relate to patient and third party privacy?

4. Is the technology cost-effective and are there any ethical obstacles to explore cost-effectiveness?

\subsection{Structural Factors with Ethical Implications:}

1. How does the technology relate to available resources in the health-care system?

2. How does the technology relate to professional values and roles? 
3. How does the technology related to specific stakeholder interests?

\subsection{Long-Term Effects:}

1. What are the potential long-term effects of the technology?

Looking at this framework in comparison with the aspects discussed in Baily et al. [1] we find a certain overlap. Questions 1 and 3 overlap with the aspects Social or scientific value and Favourable risk/benefit ratio - even if the focus is here on the actual change of care whilst the focus in Baily et al is primarily linked to QI-activities. Question 5 covers the Fair subject selection area, even if the focus in this framework is on distribution of the change or beneficial and negative effects of the change. Questions 6 and 7 are partly covered by the focus on Respect for potential and enrolled subjects and Informed consent. However, in this framework, these questions are concerned with the impact on patients and third parties when a change or improvement becomes standard care, not on the selected participants affected by specific QI-activities. From this it seems clear that the current focus on ethics in QI and focusing on the actual improvement give rise to somewhat different and complementing ethical perspectives. Still, the overlap between the two approaches imply that they cannot be used parallel with each other - rather there is need for developmental work distinguishing between how to related to ethical aspects of specific QI-activities and of the actual improvement of care. Moreover, looking at the Swedish framework, it has a definite patient (and to some extent family) focus. Given a systems and efficiency approach to QI, where an improvement might not have any real impact on patients, we probably need to further develop the framework even focusing on the actual improvement. Primarily by explicitly adding aspects related to professional health, well-being, autonomy etc. Exemplified this could imply making the following amendments.

Third party effects (question 4) should explicitly include effects on work environment for professionals, e.g. effects of work stress, work load, professional health in general. Professional values and roles (question 10) should take into account not only whether there will be a change of roles or whether there are professional values relevant for the improvement to consider - but also whether it is an ethical problem to change this role or go against these values. For example, whether the improvement will imply a change in professional autonomy, or whether professional responsibilities will change - and whether these changes are ethically warranted or problematic. The question about how an improvement relates to available resources (question 9), apart from being related to systemic effects, could also be complemented with aspects of whether there is professional competence to handle the improvement or whether there is need for professional development (with possible positive or negative effects on work environment, professional roles etc.). Hence, it is not difficult to see how this existing and implemented framework could be further developed to also cover the broader scope of quality improvement (and similarly for other existing frameworks).

As indicated in the section on HTA, describing the differences between HTA and QI, it is also important to be more observant on the specific clinical context in which the improvement is made and thus open to identify unique factors that can impact on the listed ethical values and aspects. Unfortunately, given that the tentative example below does not take place in a concrete clinical context - this can only be indicated (see question 6).

To illustrate how such a framework could be used, let us apply it to a potential quality improvement project (drawing on previous work within the field of organizational ethics) [16].

\section{ETHICAL ANALYSIS OF QUALITY IMPROVEMENT - A TENTATIVE EXAMPLE}

Within elderly care, professionals constantly phase ethical problems and conflicts [17, 18]. At the same time, at least in the Swedish context, they normally lack access to structured support systems for handling these problems and conflicts. Research shows that organized ethical reflection, and access to concrete action strategies grounded in wellestablished and relevant organizational values and norms can be supportive of professionals, and that lack of documentation and thereby, lack of organizational memory can be an obstacle in this situation [19 - 21]. Based on this, let us assume we are planning to set up a quality improvement project where we want to develop a web-based support system in which professionals can document problems and strategies, and get access to previously tried strategies, comment on and draw on these for use in their own daily work and implement this in the organization together with organized ethical reflection.

So how could this, tentatively, be analysed using the above presented framework? Following the framework, step by step, we could arrive at the following considerations: 


\subsection{Impact on Health}

1. Such an improvement is not likely to have an impact on the physical health of the elderly. However, it could be somewhat beneficial to quality of life aspects like satisfaction with care, feelings of security in facing a consistent handling of ethical conflicts arising, and indirect beneficial effects if professionals experience less ethical stress.

2. There are a number of knowledge gaps within this field, since this kind of solution has not been tested before to any large extent. However, at this point we identify no specific ethical problems in trying to fill these gaps with reference to the improvement in this case.

3. Since this is an improvement that will be used in relation to elderly care, regardless of the severity of the conditions these persons are experiencing - this question does not really apply. but generally, as elderly care at institutions is organized in Sweden today, people tend to be multi-diseased in order get access to such care and hence be on the higher end of the severity scale.

4. There are a number of third party effects to take into consideration when trying to improve elderly care this way. We know from previous studies that ethical conflicts involving significant others are common within healthcare. In a study on ethical problems within palliative care, ethical problems involving significant others constituted the main bulk of problems identified [16]. A positive effect on significant others could be a more consistent and transparent handling of ethical conflicts and problems from the professionals causing less of insecurity and more of satisfaction of care. On the negative side, this could also mean that the leeway for significant others to influence care could be more restricted than today with resulting frustration - since lack of consistent and explicit strategies can be an opening to "shop around" for preferred solutions. Hence, we need to consider whether this restriction is motivated or not. Tentatively, we might argue that lack of consistency will also enable some significant others to influence care, e.g. those with argumentative and other skills, socioeconomic power etc., but perhaps not others at the possible expense of equality and equity. As to third party effects of professionals, we know that ethical problems and conflicts might result in moral stress [18]. It is also found that organized reflection and structured ethics work can have a beneficial effect on moral stress (and other work environment factors [20].

\subsection{Specific Ethical Values and Norms}

1. As is indicated under point 4 , having a consistent and transparent approach to ethical problems can be beneficial from an equality and equity perspective. We know that intuitive decision-making in relation to ethical problems, risk being infested with prejudice and irrelevant considerations that could strengthen existing inequalities and inequities [22]. But obviously, this depends on which organizational values and norms and the strategies based on these the web-support contains. If the support is just an instrument to document existing values, norms and strategies among the professionals, existing (problematic) attitudes risk being reproduced and perhaps even more consistently applied. Hence, at this point we need to have ethical security checks of our support in order to avoid such effects. This might be about analysing the organizational values and norms fed into the system, but also about having a constant check of added strategies and comments.

2. In relation to patient and third party autonomy a number of comments can be made. As indicated in the above point about third party health effects, the resulting lack of leeway is also a restriction of the autonomy of significant others. Obviously, consistent and transparent strategies in relation to ethical problems could also restrict (some) patient's autonomy in a similar way. We then need to consider whether this is a relevant restriction and similar argument from equality and equity might be referred to as above. (For effects on professional autonomy, see below). It could be the case that in this specific clinical setting, there is a rather strong culture of paternalism of which we need to be observant and which, perhaps, should impact on what the support system emphasises but could also lead to misinterpretations of the suggested strategies on which it is important to be observant.

3. In relation to privacy, the improvement could be problematic. The documentation aspect, supposed to enable organizational memory and learning, imply that we should document situations involving both patients and significant other (and/or other professionals). Hence, we need to develop our web-support and the instructions for how to document in a way that will respect privacy. Maybe this implies having a moderating function where new posts are approved by a moderator before being published. 
4. Cost-effectiveness of the improvement is difficult to assess beforehand and will have to be assessed later on in the process.

\subsection{Structural Factors with Ethical Implications}

1. How would such a quality improvement affect available resources? First, spending time on reflection, using and documenting in the system demand resources in form of time. Resources that will have to be taken from somewhere, and we need to decide whether this is a prioritization worth doing and whether, in the long run, this could be an effective use of time (also from a resource perspective). That is, are there less important assignments which the professionals can take time from? For example, by having to spend less time on pondering frustrating and difficult ethical problems and by being better prepared to handle recurring ethical problem effectively when they arise. Here we also need to ask whether the ethical competence needed for using the support system is in place or not, or whether the quality improvement should also involve educational exercises (at a resource cost to the organization) in order for the web-support to be used.

2. From the perspective of professional values and roles, several comments can be made. First, by having a support system where organizational values and norms are explicit, and interpreted in terms of specific strategies in concrete situations - the room for idiosyncratic professional values will be restricted. At the same time, if the web-support is organized in a way to allow different strategies (implying different interpretations of the values and norms) to be documented, and allow transparent commenting on these strategies, organize group reflection where different views can be voiced - such discrepancies can be openly deliberated upon. Moreover, generally care organizations do not allow full professional autonomy when it comes to values and how they are interpreted within care practice. Hence, certain restrictions seem generally motivated. Will this improvement change the professional role? No, not really. Professional carers are expected to handle arising ethical problems and conflicts whenever they arise, and this improvement will (if successful) provide support to this task. What could happen is that the explicit reflection and documentation of ethical aspects could reveal potential ethical conflicts related to professional values and roles. For example, that different professionals interpret their ethical responsibility differently or have different ideas about central professional values. This can be challenging for the professional identity and hence we might need to be prepared to handle such reactions.

3. Generally, providing support for ethical reflection and decision-making is not likely to cause negative reactions among stakeholders - rather the opposite - even though some professional ethicists might have ideas about making ethics into "ticking a box"-exercise or trivializing ethics. However, providing explicit values, norms and strategies of the organization could arise reactions among different stakeholders. When generally accepted values and norms are explicitly interpreted into concrete strategies, this might not accord with all stakeholder interests. Hence, transparency might have a cost in terms of having to defend interpretations and strategies something that will have to be factored into the quality improvement project.

\subsection{Long-Term Effects}

1. From a long-term perspective we need to be conscious about the possible effect of this support system resulting in that professionals just engage in "rule-following" without serious reflection and thereby whether the ethical competence of the professionals will be undermined. However, if the support system builds on the idea of gathering and assessing strategies actually used by professionals and not portraying these as absolute rules such effect might perhaps be minimised. To be observent on long term effects continous assessment over time is needed.

\section{DISCUSSION}

What the above tentative example is supposed to show is how a framework for analysing health technologies could be adopted and used for quality improvements and thereby complement the current focus on ethics already suggested for QI. Even if the current ethical approach might have led to assessments in line with the above (e.g. assessing benefit/risk ratios could be given a very broad interpretation, privacy and informed consents concerns could cover also the improvement etc.), this is not the focus of the dominating approach to ethics in QI. Explicitly and transparently adding assessments of the actual improvement to the current ethics approach would therefore seem to be a quality improvement of the ethics of QI well in line with the spirit of QI. One aspect adding support to such an approach is the 
different role of informed consent suggested in Baily et al [1]. Since it is argued that the rather strict application of informed consent for participant in research is not warranted in QI, given the ethical responsibility of both professionals and patients, we need other safe-guarding instruments for what informed consent is supposed to contribute with. Apart from respecting autonomy (which might be considered a value in itself), it is also supposed to be a way to allow individual balancing of benefits and risks [23]. Such a safe-guarding mechanism then needs replacement, and an ethical assessment of the very improvement could then be part of such a replacement.

However, an expansion of the ethical assessment might be questioned for being overreaching and cumbersome for several reasons. First, in health-care research projects it is normally not required that the intervention, technology etc. to be tested is ethically assessed before the research project is terminated and the intervention or technology is to be used in health-care practice. In other words, from an ethical perspective, in research we are normally allowed the use of interventions or technologies that later could be found to be ethically problematic when implemented in practice - as long as the research project fulfils research ethical requirements. Why should we treat QI differently? The reason is the intimate connection between QI and change in practice, not at least when we are dealing with continuous QI [1]. In a traditional research project, the research phase and the implementation phase are (normally) discrete projects and distinct from each other, whereas in QI practice change (and hopefully improvement) is part and parcel of the approach. Hence, unless an ethical analysis of the intended improvement is performed before the QI is started or early in the process, it will be difficult to find a suitable place in time when to do it. Waiting and then finding it ethically problematic could prove too late, since an implemented change might be difficult to reverse.

On the other hand, this is a potential opportunity and strength of making an ethical assessment in QI instead of in HTA, i.e. that it can be sensitive to the actual characteristics of the clinical context having an impact on the relevant ethical values. This is essential to emphasise when adapting frameworks from HTA and instructions should explicitly state the need to be attentive to any unique characteristics of the clinical situation having an impact on the ethical values in question.

A (practical) problem, risk making ethical assessment of the improvement of QI cumbersome, is the idea of continuous QI. In the discussion about ethical oversight of QI, the use of existing institutional review boards (IRBs) have been criticized for this very reason. For example, in the above-mentioned Hastings Center report, it is claimed that: "Even with a more efficient IRB process, many valuable QI projects would be unable to muster the necessary resources and simply would not be done if IRB review were required. For those QI activities that did go forward, the process would impose high transaction costs on improvements that are often small in scale and represent little burden or risk to participants." [1]. Engaging a professional ethicist making an ethical analysis of the improvement before the QI-project starts might then not be an available route. What is needed is rather, ethical awareness and tools within the health-care organization to continually assess the improvements. This calls for both methods development and educational exercises within the organization. At the same time, this does not seem like an insurmountable problem. For example, the above-presented framework for identifying ethical issues is used by non-ethicists within the Swedish national HTAauthority SBU. This approach is well in line with the suggestions for ethical oversight in the Hastings Center report: "we mean that ethical oversight of QI should be fully integrated into the routine management and supervision of health care operations" [1].

Now, there might exist misconceptions about ethical analysis when expecting such an analysis to result in a yes or no to the improvement analysed. What the above tentative example of an ethical analysis of a potential quality improvement within health-care is intended to illustrate is that such an analysis could raise issues as to how such an improvement should be designed. That is, an ethical analysis is not necessarily intended to result in an assessment whether a certain improvement is ethically acceptable or not. On the contrary, few suggested improvements would result in such a conclusion from the ethical analysis (as experiences from analysing technologies within HTA shows). Rather, it identifies potential ethical weaknesses (and strengths) of the improvement, where the weakness might be handled by altering the design of the improvement or, at least, demand awareness of the possible occurrence of such a problem (and thereby also the potential of having a strategy to deal with such a problem). Once again, this is well in line with the spirit of piecemeal data-driven quality changes of QI - hence ethical assessment is simply about making the ethical assessment part of the data-set influencing improvement steps in the organization.

As indicated in the above, there are existing frameworks for ethical assessment within HTA, but to be used within QI, they need to be developed. In the tentative example some areas that need to be further developed have been indicated. Generally, this consists of aspects related to ethical considerations of how to treat health-care professionals, of how to balance between professional and patient interests from an ethical perspective, and on system considerations 
with ethical implications. Such a development could in turn inform and further sharpen frameworks used in relation to well-defined technologies within HTA. In essence, the introduction of new technologies within health-care could be viewed as a quality improvement, where considerations generally acknowledged within QI are relevant to consider in relation to all potential technologies to be used within health-care.

\section{CONCLUSION}

We have reason to expand the ethical assessment of QI to also include a more explicit ethical assessment of the actual improvement. Here existing frameworks for ethical assessment within HTA can be of use but need further development for use and integration with ethical approaches within QI. Vice versa, the introduction of new technologies within health-care could be viewed as a quality improvement, where considerations generally acknowledged within QI are relevant to consider in relation to all potential technologies to be used within health-care.

\section{CONSENT FOR PUBLICATION}

Not applicable.

\section{CONFLICT OF INTEREST}

The author declares no conflict of interest, financial or otherwise.

\section{ACKNOWLEDGEMENTS}

Declared none.

\section{REFERENCES}

[1] Baily MA, Bottrell M, Lynn J, Jennings B. The ethics of using QI methods to improve health care quality and safety. Hastings Cent Rep 2006; 36(4): S1-S40. [http://dx.doi.org/10.1353/hcr.2006.0054] [PMID: 16898359]

[2] Kass NE, Faden RR, Goodman SN, Pronovost P, Tunis S, Beauchamp TL. The research-treatment distinction: a problematic approach for determining which activities should have ethical oversight. Hastings Cent Rep 2013; 43(Spec No): S4-S15. [http://dx.doi.org/10.1002/hast.133] [PMID: 23315895]

[3] Selby JV, Krumholz HM. Ethical oversight: serving the best interests of patients. Commentary. Hastings Cent Rep 2013; 43(Spec No): S34-6. [http://dx.doi.org/10.1002/hast.138] [PMID: 23315893]

[4] Solomon MZ, Bonham AC. Ethical oversight of research on patient care. Hastings Cent Rep 2013; 43(Spec No): S2-3. [http://dx.doi.org/10.1002/hast.132] [PMID: 23315889]

[5] Jennings B, Baily MA, Bottrell M, Lynn J. Health Care Quality Improvement: Ethical and Regulatory Issues. New York: The Hastings Center 2007.

[6] Fiscella K, Tobin JN, Carroll JK, He H, Ogedegbe G. Ethical oversight in quality improvement and quality improvement research: new approaches to promote a learning health care system. BMC Med Ethics 2015; 16(1): 63. [http://dx.doi.org/10.1186/s12910-015-0056-2] [PMID: 26383770]

[7] Whicher D, Kass N, Saghai Y, Faden R, Tunis S, Pronovost P. The views of quality improvement professionals and comparative effectiveness researchers on ethics, IRBs, and oversight. J Empir Res Hum Res Ethics 2015; 10(2): 132-44. [http://dx.doi.org/10.1177/1556264615571558] [PMID: 25742674]

[8] Saarni S, Autti-Rämö I, Lühman D, et al. Ethical Analysis. HTA Core Model for Medical and Surgical Interventions 2008.

[9] Crossing the Quality Chasm: A New Health System for the 21st Century Washington. D.C.: National Academy Press 2001.

[10] Banta D. The development of health technology assessment. Health Policy 2003; 63(2): 121-32. [http://dx.doi.org/10.1016/S0168-8510(02)00059-3] [PMID: 12543525]

[11] Van der Wilt GJ, Reuzel R, Banta HD. The ethics of assessing health technologies. Theor Med Bioeth 2000; 21(1): 103-15. [http://dx.doi.org/10.1023/A:1009934700930] [PMID: 10927971]

[12] Hofmann B. Toward a procedure for integrating moral issues in health technology assessment. Int J Technol Assess Health Care 2005; 21(3): 312-8. [http://dx.doi.org/10.1017/S0266462305050415] [PMID: 16110710]

[13] Autti-Rämö I, Mäkelä M. Ethical evaluation in health technology assessment reports: an eclectic approach. Int J Technol Assess Health Care 2007; 23(1): 1-8.

[http://dx.doi.org/10.1017/S0266462307051501] [PMID: 17234010]

[14] Effekter av individuell samordnare av vård- och omsorgsinsatser för de mest sjuka äldre - en systematisk översikt (Effects of individual coordinator of care for multi-diseased elderly - a systematic review). Stockholm: SBU 2015. 
[15] Heintz E, Lintamo L, Hultcrantz M, et al. Framework for systematic identification of ethical aspects of healthcare technologies: the SBU approach. Int J Technol Assess Health Care 2015; 31(3): 124-30. [http://dx.doi.org/10.1017/S0266462315000264] [PMID: 26134927]

[16] Sandman L, Molander U, Benkel I. Developing organisational ethics in palliative care: A three-level approach. Nurs Ethics 2015; 24(2): 138-50.

[17] Bolmsjö IA, Sandman L, Andersson E. Everyday ethics in the care of elderly people. Nurs Ethics 2006; 13(3): $249-63$. [http://dx.doi.org/10.1191/0969733006ne875oa] [PMID: 16711184]

[18] Juthberg C, Eriksson S, Norberg A, Sundin K. Perceptions of conscience, stress of conscience and burnout among nursing staff in residential elder care. J Adv Nurs 2010; 66(8): 1708-18 [http://dx.doi.org/10.1111/j.1365-2648.2010.05288.x] [PMID: 20557396]

[19] van der Dam SS, Abma TA, Molewijk AC, Kardol MJ, Schols JM, Widdershoven GA. Organizing moral case deliberation experiences in two Dutch nursing homes. Nurs Ethics 2011; 18(3): 327-40. [http://dx.doi.org/10.1177/0969733011400299] [PMID: 21558109]

[20] van der Dam S, Schols JM, Kardol TJ, Molewijk BC, Widdershoven GA, Abma TA. The discovery of deliberation. From ambiguity to appreciation through the learning process of doing Moral Case Deliberation in Dutch elderly care. Soc Sci Med 2013; 83: 125-32. [http://dx.doi.org/10.1016/j.socscimed.2013.01.024] [PMID: 23410684]

[21] Dauwerse L, van der Dam S, Abma T. Morality in the mundane: specific needs for ethics support in elderly care. Nurs Ethics 2012; 19(1): 91-103. [http://dx.doi.org/10.1177/0969733011412102] [PMID: 22140180]

[22] Kahneman D. Thinking, fast and slow. New York: Farrar, Straus and Giroux 2011.

[23] Taylor JS. Autonomy and informed consent: A much misunderstood relationship. J Value Inq 2004; 38(3): $383-91$. [http://dx.doi.org/10.1007/s10790-005-5868-8]

\section{2017 Lars Sandman.}

This is an open access article distributed under the terms of the Creative Commons Attribution 4.0 International Public License (CC-BY 4.0), a copy of which is available at: https://creativecommons.org/licenses/by/4.0/legalcode. This license permits unrestricted use, distribution, and reproduction in any medium, provided the original author and source are credited. 